\title{
PREPARATION OF CARBAMAZEPINE CHITOSAN NANOPARTICLES FOR IMPROVING NASAL ABSORPTION
}

\author{
*Arya Rajeshwar Kamal Kant, Juyal Vijay, Kunwar Nitin \\ Department of Pharmaceutical Sciences, Bhimtal Campus Kumaun University, Nainital, India \\ *Corresponding Author's E-mail Id: rajeshwararya@gmail.com
}

\begin{abstract}
In this study the nasal administration of carbamazepine has been studied using chitosan nanopartcles. The chitosan nanoparticles were prepared by ionic gelation of chitosan $(100-300 \mathrm{mg})$ with Tripolyphosphate sod (100-300mg in $100 \mathrm{ml}$. Nine formulations were prepared, characterized and compared in terms of morphology (Transmission electron microscopy), drug content, particle size (zetasizer) and In-vitro drug release. In-vitro drug release studies were performed in Franz diffusion cell using phosphate buffer (buffer $\mathrm{pH} 5.5$ ) as dissolution medium. The Chitosan nanoparticles had a mean size of $124.2 \pm 05$ to $580 \pm 13 \mathrm{~nm}$, zeta potential were found to be +21 to $26.6 \mathrm{mV}$ and the entrapment efficiency were found to be 65 to $72.7 \%$. The in-vivo study was performed on Wistar rat, nanoparticles were administered through nasal route and compared with carbamazepine given by i.v. route, the results indicate that carbamazepine loaded chitosan nanoparticles enhances the drug absorption through nose. The results showed that the carbamazepine could be directly transported into the rat brain through nose and the possible side effects could be minimized.
\end{abstract}

Key Words: Carbamazepine, Chitosan Nanopartcles, Ionic Gelation, Epilepsy

\section{INTRODUCTION}

Epilepsy is a common neurological disorder affecting approx. $0.5-1 \%$ population in all over the world. There are several formulations in the market those are employed for the treatment of epilepsy. Antiepileptic drug after oral or intravenous administration exhibits high distribution of drug into brain and other nontargeted organs which can precipitate the undesirable side effects e.g. somnolence, dizziness and ataxia to severe peripheral pathologic conditions like dermatologic and hematologic, renal and hepatic dysfunctions. ${ }^{1,2,3}$ The prevention of such kind of condition is very difficult but a much needed work, for this the drug should be targeted directly into the brain. But to deliver the drugs directly into the brain is a very difficult task because the penetration of drugs into brain is a great challenge, due to the functionality and structure of the BBB. The blood brain barrier is a unique system of capillary endothelial cell which prevent foreign material to permeate into the brain. BBB limits the transport of drugs by tight junction or physical barrier and the transendothelial electron resistance (TEER), enzymes or metabolic barrier also limits the penetration into brain. ${ }^{4}$ In last 10 years, different techniques have been attempted to target the brain to deliver drugs directly into the brain. Nowadays alternative route of drug delivery is in practice, nasal route has attracted scientist's attention to develop new drug delivery system. ${ }^{5}$ Nasal route appears to be an ideal alternative to parenterals for systemic drug delivery as well as for brain tageting. ${ }^{6}$ Nasal drug delivery is a noninvasive method and it also avoids gastrointestinal and hepatic first-pass metabolism. It exhibit rapid-onset of action, patient compliance, self administrable, which make it a route of choice for the management of emergency situations. ${ }^{7}$ Despite these advantage nasal drugs delivery has some limitations for drug penetration like size, molecular weight and low residence time of drugs in the nasal cavity. ${ }^{4,7}$ To overcome these problems particulate carrier like nanoparticles made up of mucoadhesive biomaterial can be used to target brain via nasal route due to their small size and mucoadhesive properties. ${ }^{8}$ Polymeric nanoparticles are solid colloids having a size of $10-1000 \mathrm{~nm}$. Chitosan is widely used polymer for preparation of mucoadhesive nanoparticles. Chitosan shows very good entrapment of drugs. Various studies have shown the successful use of chitosan nanoparticles for better drug targeting in brain as well as systemic circulation. ${ }^{8}$

Carbamazepine is first-line antiepileptic drugs with narrow therapeutic window, complex pharmacokinetics, it has chances of interacting with other drugs and causing side effects, it is absorbed slowly from the oral route, its oral bioavailability is $75 \%$ and the peak plasma concentration is achieved in about $4-8 \mathrm{~h}$ after dosing, it may be vary to $24 \mathrm{~h}$ at high doses. ${ }^{2}$ Carbamazepine metabolized by liver and the enzymatic induction can 
cause the unpredictable fluctuation in plasma and an increase in unexpected clearance. For this the dose adjustment is required. All these pharmacokinetics limitation of orally administered carbamazepine make it, a suitable candidate for making nanoparticles using chitosan polymer for nasal administration.

\section{MATERIALS AND METHOD}

\subsection{Material}

Chitosan was obtained as a gift sample from CIFT, Kocchi, and Carbamazepine was purchased from Sigma Alderich Mumbai, Tween80 was purchased from Central Drug House, Mumbai, Dialysis membrane-70 was purchased from Hi Media, Mumbai. All other chemicals and solvents used in the study were of analytical or HPLC grade.

\subsection{Method}

Nanoparticles were prepared by using ionic gelatin technique, which was described by Calvo, ${ }^{11}$ in which $100-300 \mathrm{mg}$ chitosan was dissolved in $100 \mathrm{ml}$ aq. solution of $0.5 \%$ acetic acid. The aq. solution of 100 $300 \mathrm{mg}$ TPP sod was prepared separately. Then the required amount of drug was dissolved in $10 \mathrm{ml}$ methanol and mixed into chitosan solution. Then $25 \mathrm{ml}$ of the aq. solution of TPP sod was added to $100 \mathrm{ml}$ of chitosan solution and stirred for $2 \mathrm{hr}$ at $4000 \mathrm{rpm}$ using Remi mechanical stirrer. The formed nanoparticles were collected by centrifuging the system for $10 \mathrm{~min}$ at $5000 \mathrm{rpm}$ and then air dried. Nine formulations were prepared by varying chitosan and TPP sod conc.

\subsubsection{Particle size:}

The average diameter and Polydispersity Index (PDI) of prepared batches of carbamazepine loaded chitosan nanoparticles were determined by Photon Correlation Spectroscopy (PCS) using a Zetasizer (Malvern, Ver. 6.01) at a fixed angle at $25^{\circ} \mathrm{C}$. Sample was diluted 10 times with distilled water and then it was analyzed for particle size. The readings were recorded in triplicate.

\section{2.2 Zeta potential:}

The zeta potential can be measured by determination of the movement velocity of the particles in an electric field and the particle charge, the chitosan nanoparticles dispersion was diluted 10 times with distilled water and analyzed by Zetasizer (Malvern, Ver. 6.01).

\subsubsection{Drug Entrapment Efficiency}

The entrapment efficiency (EE), which corresponds to the percentage of carbamazepine encapsulated within and adsorbed on to the nanoparticles, was determined by measuring the concentration of free carbamazepine in the dispersion medium. $10 \mathrm{mg}$ of carbamazepine loaded nanoparticles was dissolved into $10 \mathrm{ml}$ methanol and kept for overnight then centrifuged at 5000rpm for $10 \mathrm{~min}$. Supernatant was than filtered by $0.2 \mu$ membrane filter and analyzed by UV-VIS spectroscopy at 280.4 $\mathrm{nm}$.

$$
\% \mathrm{EE}=\frac{[\text { Initial drug }- \text { Free drug }] \times 100}{\text { Initial drug }}
$$

Where, Initial drug is the mass of initial drug used for the assay.

Free drug is the mass of free drug detected in the supernatant after centrifugation of the aqueous dispersion.

\subsubsection{Drug Content}

$10 \mathrm{mg}$ of carbamazepine nanoparticles was dissolved into $10 \mathrm{ml}$ methanol and kept for overnight. The soaked solution was centrifuged at $5000 \mathrm{rpm}$ for $10 \mathrm{~min}$ to separate the polymer. Supernatant was than filtered by $0.2 \mu$ membrane filter and analyzed by UV-VIS spectroscopy at $280.4 \mathrm{~nm}$.

\subsubsection{In-Vitro Drug Release Study nanoparticles ${ }^{12}$}

The in-vitro release study was performed using previously overnight soaked dialysis membrane-70 mounted over the receptor compartment of Franz diffusion cell. The donor compartment was fixed and $37^{\circ} \mathrm{C} \pm 0.5^{\circ} \mathrm{C}$ and stirred at $100 \mathrm{rpm}$ with Teflon-coated magnetic stirring bars. The formulations were placed in donor compartment and dialysis medium (buffer $\mathrm{pH}$ 5.5) was filled in receptor compartment. At predetermined time intervals $(0.5,1,2,3$, for $9 \mathrm{hrs}$.), $5 \mathrm{ml}$ aliquots was withdrawn and replaced with the same amount of fresh medium. Sink condition was maintained throughout the experiment. The amount of carbamazepine released from the nanoparticles was measured by UV spectrophotometer at $280.4 \mathrm{~nm}$.

\subsubsection{In-Vitro Drug Release Kinetics ${ }^{13,14}$}

In order to investigate the mechanism of release, the release data were analyzed with the following mathematical models: zero-order kinetic, first-order kinetic, Korsmeyer-Peppas kinetic model and Higuchi kinetic model.

\subsubsection{Pharmacokinetic studies ${ }^{1}$}

The rats were divided into two groups (A and B), Group A containing 18 animals which received the drug by i.v. administration and group B containing 18 animals received nanoparticles by nasal route. At a set time interval $(5,15,30,45,60,120 \mathrm{~min})$ after dosing 3 animal per time point were sacrificed by cervical dislocation then decapitated. The blood was then immediately collected in tube containing heparin. The brain was removed and weighed. The blood sample were centrifuged at $4^{0} \mathrm{C}$ at $4000 \mathrm{rpm}$ for $10 \mathrm{~min}$. the plasma were stored at $-300 \mathrm{C}$ for further analysis. The brain was homogenized with $0.1 \mathrm{M}$ sod phosphate buffer pH5.0 (4ml per gram) of tissue. The Teflon pestle tissue homogenizer was used. Tissue homogenate was then centrifuged at $4000 \mathrm{rpm}$ for $15 \mathrm{~min}$ at $4^{0} \mathrm{C}$ and the supernatant was then kept at $-300 \mathrm{C}$ for further analysis.

\subsubsection{In-vivo experiments in rat}

The in-vivo experiments were performed on Wistar rat at Deshpande Lab Bhopal, India. All animal studies were performed according to CPCSEA. The CPCSEA/IAEC approval No. IAEC /DL/ 2015/RK/012. 


\subsubsection{Preparation of IV Drug solution for i.v. Administration}

For i.v. administration, the drug was dissolved in a mixture of propylene glycol-physiologic saline $(0.9 \% \mathrm{NaCl})$-ethanol in a ratio of $5: 3: 2$ to make a final conc. of $1 \mathrm{mg}$ per $\mathrm{ml}$

\subsubsection{Intranasal and i.v. administration ${ }^{1}$}

The rats were anaesthetized with an intraperitoneal injection of a mixture of ketamine $(100 \mathrm{mg} / \mathrm{kg}$ ) and xylazine $(10 \mathrm{mg} / \mathrm{kg})$ and the temperature of room was maintained warm. The prepared i.v. solution at dose of $0.1 \mathrm{mg} / \mathrm{kg}$ was administered by injection on lateral tail vein. The prepared nanoparticles were administered at a dose equivalent to $1 \mathrm{mg} / \mathrm{kg}$ to rat. The rat was placed on one side and the formulation was instilled using a polyurethane tube attached to a syringe. The tube inserted to $10 \mathrm{~mm}$ deep into one the nares, to deliver the formulation to roof of the nasal cavity.

\subsubsection{Drug Analysis:}

Determination of drug was performed by a liquid chromatography system equipped with DAD detector 1100 at $285 \mathrm{~nm}$ (Agilent Technologies, Milano, Italy). A Luna ${ }^{\circledR}$ phenyl hexyl 5-m (Phenomenex, Torrance, USA) $250 \mathrm{~mm} \times 4.6 \mathrm{~mm}$ column with cartridge precolumn was used for separation at a flow rate of $0.60 \mathrm{ml} / \mathrm{min}$. The mobile phase consisted of acetonitrile, methanol and water $(0.1 \%)$ 30:60:10 (v/v) and the injection volume was $100 \mu 1$. The amount of carbamazepine in serum and brain was expressed as $\mathrm{ng} / \mathrm{ml}$ serum.

\subsubsection{Pharmacokinetic analysis ${ }^{13}$}

The peak plasma conc. $\mathrm{C}_{\max }$ of drug was directly observed from plasma or brain and the time $\left(\mathrm{T}_{\max }\right)$ to reach $\mathrm{C}_{\max }$ was directly estimated from the data received by experiments, other Pharmacokinetic parameter were calculated based on the SEM $(n=3)$ at each time point by a non compartment pharmacokinetics analysis. The pharmacokinetics parameters like AUC were evaluated, from $t_{0}$ to the last quantifiable conc. $t_{\text {last }}$ by linear trapezoidal rule.

To assess the brain targeting efficiency of nasal formulations the drug targeting efficiency (DTE) index was calculated. It is a ratio of nasal and i.v.

$$
D T E=\frac{(\text { AUC brain/AUCplasma }) \text { nasal }}{(\text { AUC brain/AUCplasma }) \text { iv }}
$$

Where AUCbrain and AUCplasma are the area under the drug conc. time curve for brain and plasma after nasal and i.v. administration. For an effective drug targeting the ratio should be more than one.

\subsubsection{Statistical analysis}

The data were expressed as SEM, the comparative studies done between i.v. and nasally delivered formulation using single unpaired one tail ANOVA, difference was considered significant for a $\mathrm{p}$ - value $\mathrm{p}<0.05$.

\section{RESULT AND DISCUSSION}

\subsection{Preparation of Chitosan Nanoparticles}

In present study, ionic gelation method was used to prepare nanoparticles, a simple and reproducible method for preparation of polymeric nanoparticles

\subsection{Characterization of Chitosan Nanoparticles:}

\subsubsection{Particle Size:}

In all the formulations, the particles size ranged from $124.2 \pm 05$ to $580 \pm 13 \mathrm{~nm}$, and polydispersibility index were found to be $0.261 \pm 0.15$ to $0.656 \pm 0.073$. The results are given in table no.1. The study reveals that, polymer conc. have a significant effect on size, at the lower conc. of polymer, small size nanoparticles formed, but on increasing the conc. of polymer the size of nanoparticles increased, at higher conc. the chitosan forms a viscous gel which would not break down into small particles at given stirring speed, therefore a high particle size was observed. The conc. of sod TPP also plays effective role in size controlling, as the conc. of TPP sod was increased the particles size was decreased.

\subsubsection{Zeta Potential}

In all the formulations, the zeta potential ranged from +32.1 to +15.1 . The chitosan is positively charged polymer therefore as the conc. of chitosan increased the zeta potential increases, and because the TPP sod is negatively charged therefore on increasing the conc. of TPP sod the zeta potential decreased. The results are given in table no. 1

The higher zeta potential shows that all formulation are stable because the high zeta potential would not allow the particles to get aggregate or to form a big colloid due to electrical repulsion between particles. The Tween-80 would also provide stabilization to the formulations. The final results are shown in table no. 1 
Results

$\begin{array}{rlllll} & & \text { Mean }(\mathrm{mV}) & \text { Area }(\%) & \text { Width }(\mathrm{mV}) \\ \text { Zeta Potential }(\mathrm{mV}): & 25.4 & \text { Peak 1: } & 25.4 & 100.0 & 8.25 \\ \text { Zeta Deviation }(\mathrm{mV}): & 8.25 & \text { Peak 2: } & 0.00 & 0.0 & 0.00 \\ \text { Conductivity }(\mathrm{mS} / \mathrm{cm}): & 0.00833 & \text { Peak 3: } & 0.00 & 0.0 & 0.00 \\ \text { Result quality : Good } & & & & \end{array}$

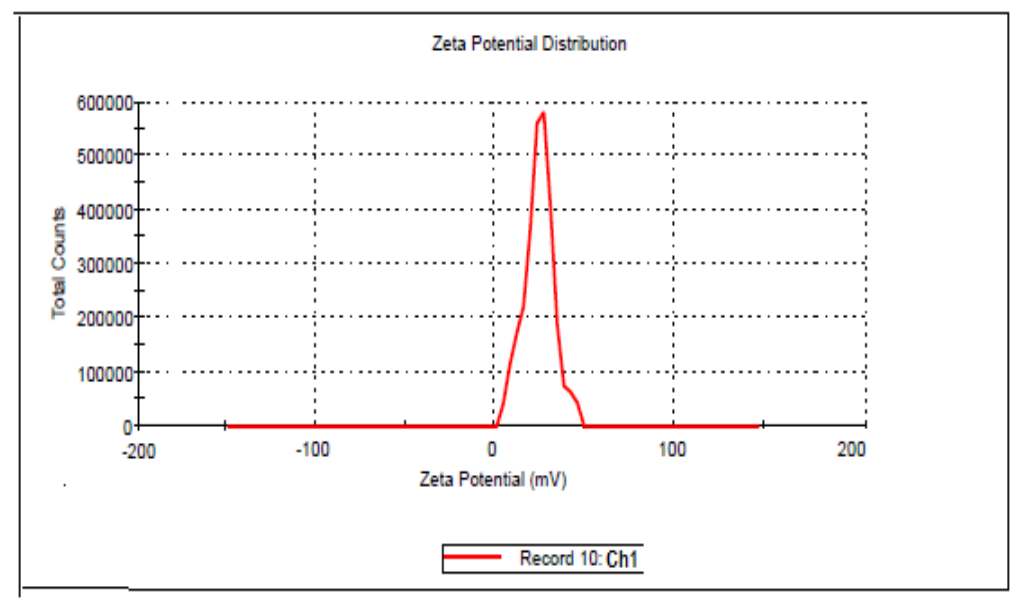

Figure 1: showing particle size of formulation Ch1

$\begin{array}{rlllll} & & \text { Diam. }(\mathrm{nm}) & \text { \% Intensity } & \text { Width }(\mathrm{nm}) \\ \text { Z-Average (d.nm): } 124.2 & \text { Peak 1: } & 86.15 & 52.1 & 34.48 \\ \text { Pdl: } 0.458 & \text { Peak 2: } & 404.3 & 46.8 & 193.5 \\ \text { Intercept: } 0.965 & \text { Peak 3: } & 5174 & 1.1 & 488.5 \\ \text { Result quality: } & & & & \end{array}$

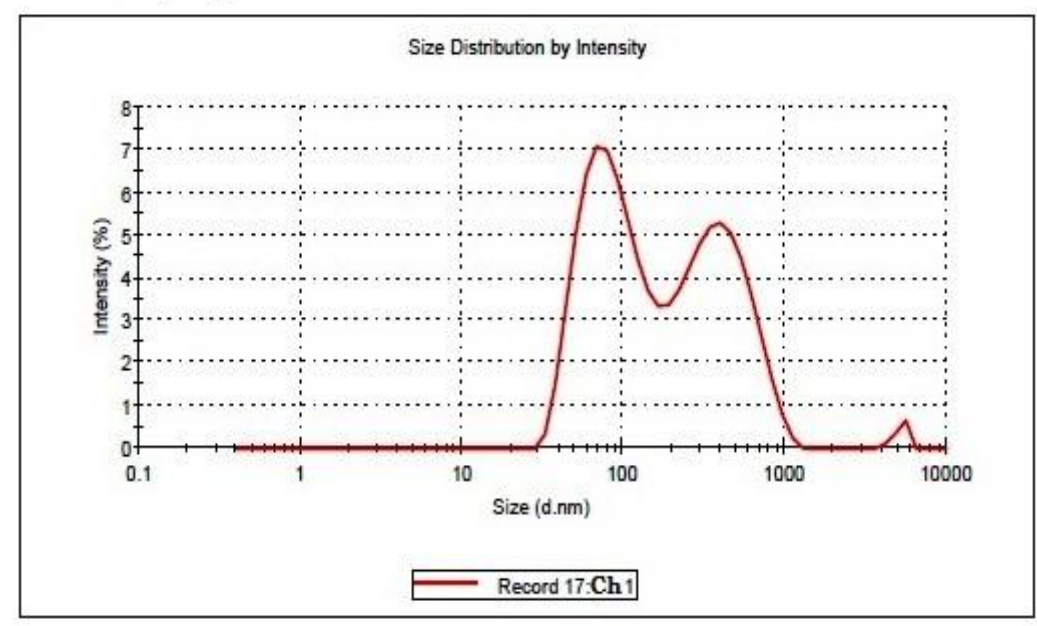

Figure 2: showing zeta potential of formulation Ch1

\subsubsection{Drug Entrapment Efficiency}

Percentage entrapment efficiency of all the formulations was found to be $40 \pm 1.25$ to $69 \pm 0.85$. The results are given in table no. 1

The results shows that the polymer conc. play an important role in drug entrapment, on increasing the conc. of chitosan initially favors increase in the entrapment efficiency up to a certain level and then a decrease in the entrapment efficiency was observed may be due to the formation of viscous gel at higher polymer conc. i.e. $0.3 \%$, the gel might hinder the permeation of drug into chitosan polymer matrix during formation of nanoparticles.

The conc. of TPP sod also affects the entrapment efficiency, at higher conc. of TPP sod the entrapment efficiency also decreased due to formation of a cross linked network which would not allow the drug to get diffuse into the polymer-TPP network.

\subsubsection{Drug Content}

Percentage drug content of all the formulation was found to be $84.69 \%$ to $98.85 \%$. The final results are shown in table no. 1 
Table 1:

\begin{tabular}{|c|c|c|c|c|c|c|}
\hline S.NO. & Batch code & Zeta size (nm) & PDI & $\begin{array}{ll}\begin{array}{l}\text { Zeta } \\
(\mathrm{mV})\end{array} & \text { potential } \\
\end{array}$ & $\% \mathrm{EE}$ & $\begin{array}{l}\text { \%Drug } \\
\text { content }\end{array}$ \\
\hline 1 & Ch1 & $124.2 \pm 05$ & $0.368 \pm 0.12$ & +25.4 & $65 \pm 0.12$ & $84.69 \%$ \\
\hline 2 & Ch2 & $370 \pm 07$ & $0.258 \pm 0.154$ & +28.9 & $69 \pm 1.02$ & $98.85 \%$. \\
\hline 3 & Ch3 & $580 \pm 13$ & $0.431 \pm 0.09$ & +32.1 & $56 \pm 0.98$ & $88.34 \%$ \\
\hline 4 & Ch4 & $108 \pm 10$ & $0.368 \pm 0.65$ & +17.2 & $58 \pm 0.56$ & $88.25 \%$ \\
\hline 5 & Ch5 & $310 \pm 05$ & $0.261 \pm 0.15$ & +19.2 & $61 \pm 0.80$ & $86.05 \%$ \\
\hline 6 & Ch6 & $410 \pm 09$ & $0.461 \pm 0.106$ & +20.1 & $51 \pm 1.07$ & $85.85 \%$ \\
\hline 7 & Ch7 & $89 \pm 03$ & $0.638 \pm 0.170$ & +15.1 & $48 \pm 1.10$ & $90.85 \%$ \\
\hline 8 & Ch8 & $250 \pm 10$ & $0.656 \pm 0.073$ & +17.5 & $53 \pm 0.85$ & $92.85 \%$ \\
\hline 9 & Ch9 & $325 \pm 12$ & $0.361 \pm 0.08$ & +19.8 & $40 \pm 1.25$ & $91.85 \%$ \\
\hline
\end{tabular}

\subsubsection{In-vitro drug release}

The in-vitro cumulative drug release was found to be $69.8 \%$ to $82.8 \%$. The formulation $\mathrm{Ch} 2$ shows the highest drug release in $9 \mathrm{hr}$ i.e. $86.3 \%$ where as $\mathrm{Ch} 9$ shows the lowest drug release i.e. $69.8 \%$ in $9 \mathrm{hr}$. it was observed in the study that the conc. of chitosan and the conc. of TPP sod plays important role in drug release from the formulations. Initially in the first $120 \mathrm{~min}$ the drug released highest from the $\mathrm{Ch} 3$ having the highest conc. of chitosan but after $120 \mathrm{~min}$ the release is retarded because its forms gel, which retarded the drug release. On the other hand the increase in TPP sod conc. also retarded drug release because higher conc. of TPP sod makes a close network within the chitosan polymeric network.

\subsubsection{Effect of chitosan conc. on drug release}

Statistical analysis was used to analyze and check, whether there was any significant difference present in drug release pattern of different formulations or not. The single factor ANOVA was done with the help of MS Excel 2007 Software for a time period of 9hr applied for drug release, with increase in conc. of chitosan, a significant decrease $p<0.05(0.009335)$ was observed in in-vitro release of carbamazepine from nanoparticles, as the conc. of chitosan increased from $0.1 \%$ to $0.2 \%$ the drug release increased from $82.8 \%$ to 86.3 but on increasing the conc. of chitosan to $0.3 \%$ from $0.2 \%$ the drug release was decreased.

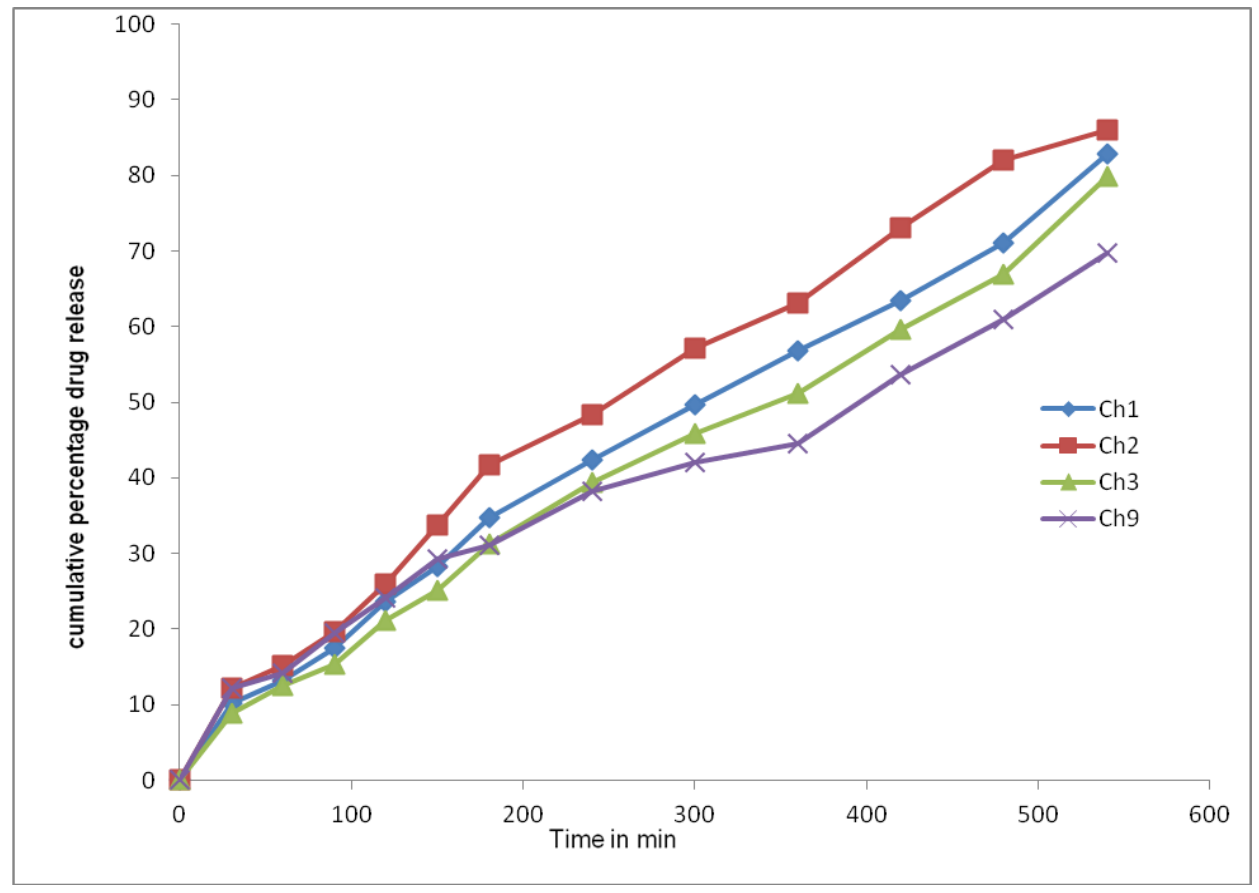

Figure 3: showing In-vitro drug release of formulation $\mathrm{Ch} 1, \mathrm{Ch} 2, \mathrm{Ch} 3$ and $\mathrm{Ch} 9$

In first $2 \mathrm{hr}$, formulation $\mathrm{Ch} 3 \mathrm{has}$ higher chitosan conc. shows faster release about $31.3 \%$ than $\mathrm{Ch} 1$ and $\mathrm{Ch} 2$ but the drug release then decreased in comparison to $\mathrm{Ch} 1$ and $\mathrm{Ch} 2$. This is may be due to the formation of gel at higher chitosan conc.

\subsubsection{Effect of cross linking agent}

To understand the effect of cross linking agent on the drug release, formulation $\mathrm{Ch} 1, \mathrm{Ch} 2$ and $\mathrm{Ch} 3$ were analyzed with increasing conc. of TPP sod a significant 
$\mathrm{p}<0.05(0.006163)$ decrease in release rate was observed which revealed that the higher conc. of cross linking agent retards the drug release. The $80.7 \%, 84.23 \%$ and $86.51 \%$ carbamazepine was released from $\mathrm{Ch} 1, \mathrm{Ch} 2$ and $\mathrm{Ch} 3$ respectively.

\subsubsection{Kinetics modeling of all formulation}

The drug release kinetics of all formulations was evaluated to understand the drug release behavior from the chitosan nanoparticles. The drug release data were evaluated for a best fit equation of Zero order, First order, Koresymer Papas and Higuchi model. From the above study, the drug release data of most of the formulations fits into Korsmeyer Papps model where $r^{2}$ value were found near to 0.999 , for the drug release of formulations Ch1, Ch2, Ch3, Ch4, Ch6 and Ch9. The formulations $\mathrm{Ch} 5, \mathrm{Ch} 7$, and $\mathrm{Ch} 8$ have shown a zero order drug release. But the drug release data of formulation Ch1 has shown a perfect Korsmeyer Papps model where the $r^{2}$ value was found to be 0.9904 and the
$\mathrm{K}$ value was found to be 0.6059 , which shows that the drug release follows Korsmeyer Papps model with nonFickian diffusion super class II $(0.45<\mathrm{n}<0 \quad 8.9)$. The drug release from the chitosan nanoparticles may be due to the diffusion of water into the nanoparticles followed by swelling and finally formation of gel. The nonfickian case-II transport mechanism associated with formation of water soluble glassy polymer. The first $60 \%$ drug release data fits into Korsmeyer Papps from the above studies formulation $\mathrm{Ch} 1$ is selected for further study because it has narrow size range $124.2 \mathrm{~nm}$ with good entrapment efficiency $65 \%$ and a good release profile. In this formulation the drug release initially showed a burst release and then a constant release for a period of $9 \mathrm{hr}$.

\subsubsection{Transmission Electron Microscopy:}

The TEM study of the optimized formulation Ch1 was done, which also confirms the size of the prepared nanoparticles.

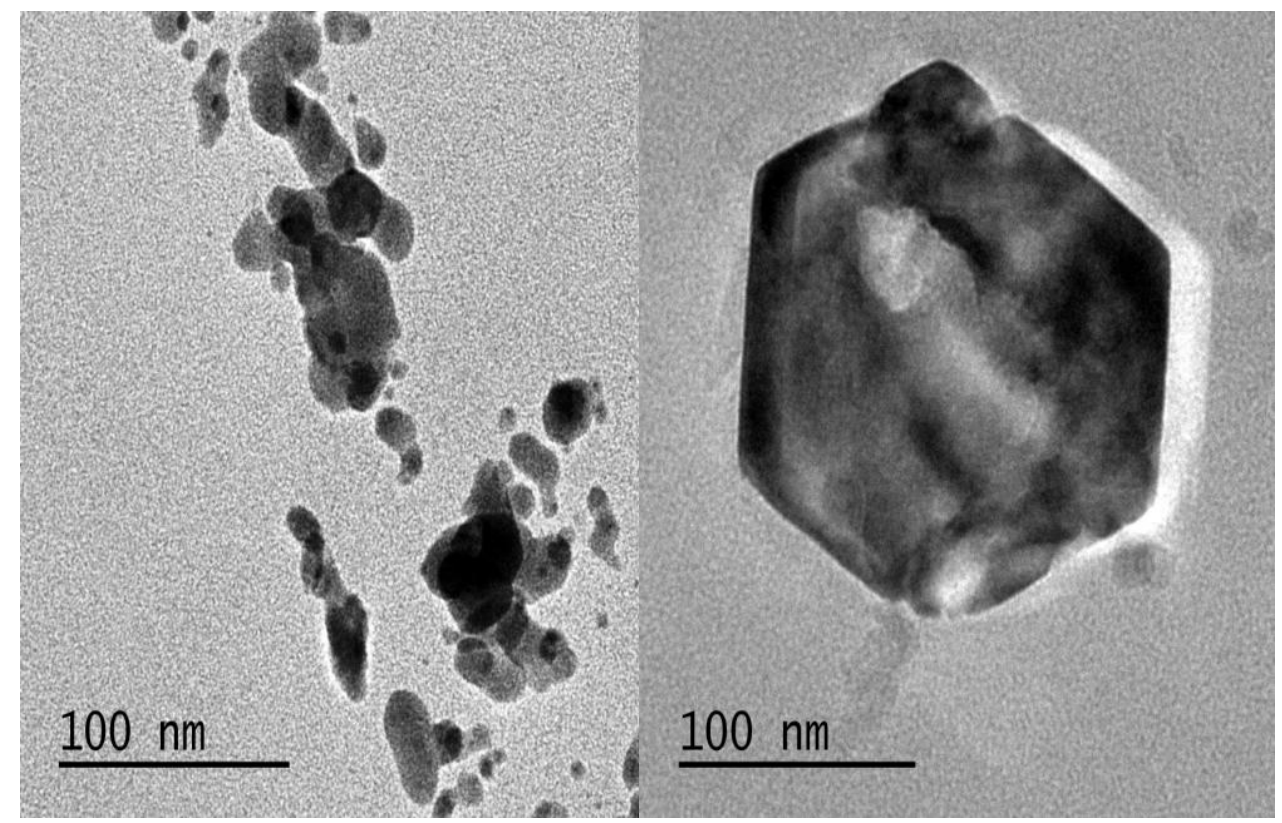

Figure 4: Showing TEM photograph (A) of Formulation Ch1 and B showing single particle of Formulation Ch1

\subsubsection{Pharmacokinetics study of drug from i.v. and nasally applied chitosan nanoparticles $\mathrm{Ch1}$}

The pharmacokinetic data from i.v. administration, the $\mathrm{C}_{\max }$ and $\mathrm{T}_{\max }$ in plasma and brain was directly determined, in first $5 \mathrm{~min}$ the $\mathrm{C}_{\max }$ was achieved in plasma but in brain it has taken $30 \mathrm{~min}$ to achieve $\mathrm{C}_{\max }$. The AUC in plasma and brain was calculated by trapezoidal method, the i.v. AUC(Plasma) $)_{0-120 \text { min }}$ was found to be $110.51 \mu \mathrm{gml}^{-1} \mathrm{~min}^{-1}$ and $\mathrm{AUC}(\text { Brain })_{0-120 \mathrm{~min}}$ was found to be $78.95 \mu \mathrm{gml}^{-1} \mathrm{~min}^{-1}$. The $\mathrm{C}_{\max }$ in plasma 1199 ng and brain was found to be $1357 \mathrm{ng}$. The ratio of AUC(Brain) and AUC(Plasma) was found as 0.7144, which shows, the distribution of drug into brain (targets) and plasma (non target) slightly equal. The study reveals that the conc. in plasma is initially higher, this higher conc. can precipitate the side effects, after some time the conc. was found higher in brain than plasma.
Then the formulation Ch1 was given nasally to Wister rat after a single dose of drug $1 \mathrm{mg} / \mathrm{kg}$, the corresponding pharmacokinetic parameter estimated by noncompartmental analysis and AUC was calculated by trapezoidal method, initial conc. was extrapolated to the origin. In first $5 \mathrm{~min}$ the $\mathrm{C}_{\max }$ was achieved in brain but in plasma it has taken $45 \mathrm{~min}$ to achieve $\mathrm{C}_{\max }$. The AUC (Plasma) 0-120min was found to be $104.43 \mu \mathrm{gml}^{-1} \mathrm{~min}^{-1}$ and AUC (Brain) 0-120min was found to be $129.53 \mu \mathrm{gml}^{-1} \mathrm{~min}^{-}$ 1 . The $\mathrm{C}_{\max }$ in plasma 1430ng and brain was found to be 2920ng. The ratio of AUC (Brain) and AUC(Plasma) was found as 1.240 , which shows, the high distribution into brain than plasma can be achieved with chitosan nanoparticles.

The drug transport efficiency was found to be 1.735 , which shows that a chitosan nanoparticle has good brain targeting efficiency. 


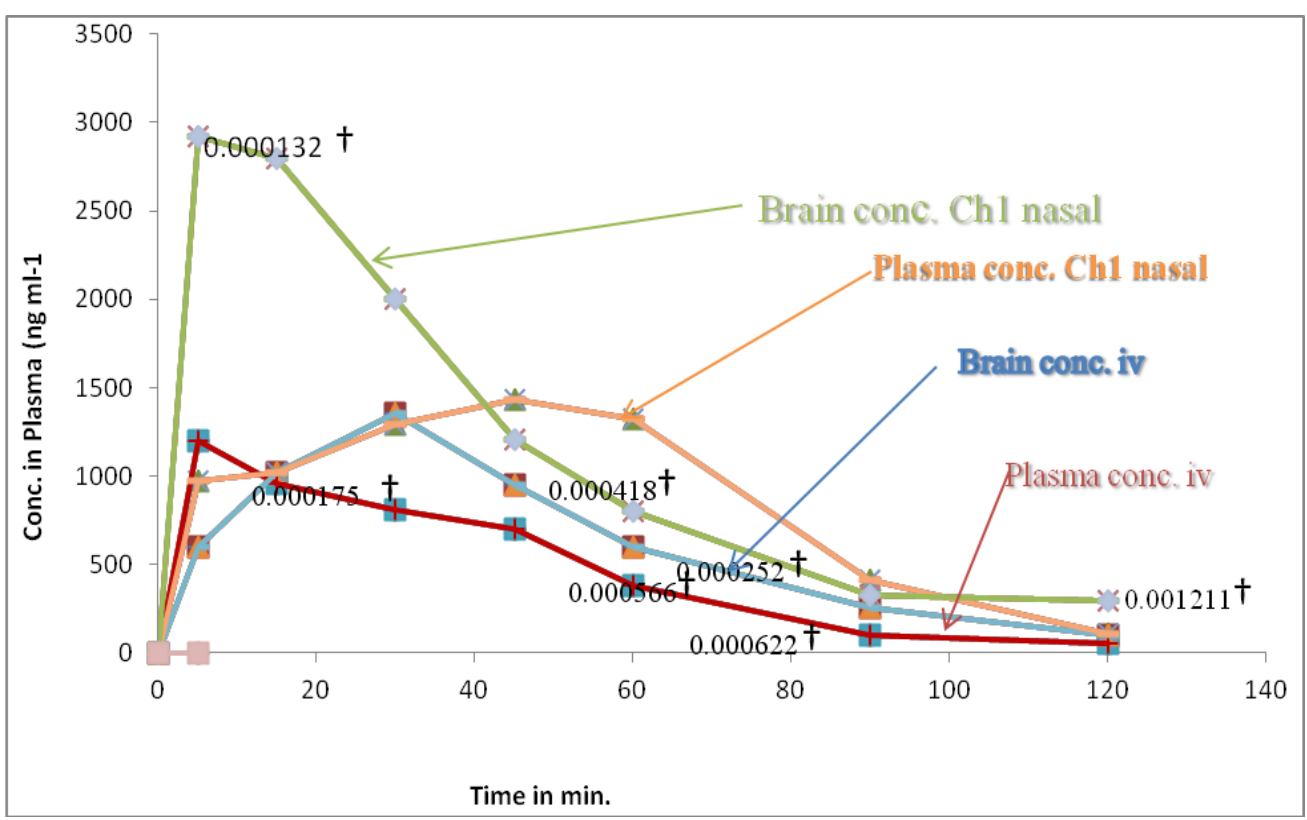

Figure 5: showing plasma and brain concentration- time profile of carbamazepine after i.v and nasally applied nanoparticles, The results are mean values \pm SEM, $n=3, \uparrow$ represents significant difference at any time point.

Table 2: Showing Pharmacokinetic parameters in brain and plasma after nasal and i.v. administration

\begin{tabular}{|c|c|c|c|c|}
\hline Route & Organ/tissue & $\mathrm{C}_{\text {Max }}$ & $\mathbf{T}_{\text {Max }}$ & $\mathrm{AUC}_{0-120 \min }$ \\
\hline \multirow[t]{2}{*}{ i.v. } & Plasma & 1199 ng & $5 \mathrm{~min}$ & $110.51 \mu \mathrm{gml}^{-1} \mathrm{~min}^{-1}$ \\
\hline & Brain & $1357 \mathrm{ng}$ & $30 \mathrm{~min}$ & $78.95 \mu \mathrm{gml}^{-1} \mathrm{~min}^{-1}$ \\
\hline \multirow[t]{2}{*}{ Nasal } & Plasma & 1430ng & $45 \mathrm{~min}$ & $104.43 \mu \mathrm{gml}^{-1} \mathrm{~min}^{-1}$ \\
\hline & Brain & 2920ng & $5 \mathrm{~min}$ & $129.53 \mu \mathrm{gml}^{-1} \mathrm{~min}^{-1}$ \\
\hline AUC (Brain) /AUC(Plasma) nasal & 1.240 & & & \\
\hline AUC (Brain) /AUC (Plasma) i v & 0.7144 & & & \\
\hline DTE & 1.735 & & & \\
\hline
\end{tabular}

\section{SUMMARY AND CONCLUSION}

The chitosan nanoparticles of carbamazepine were successfully prepared by ionic gelation method using chitosan as polymer and TPP sod as ionic gelation agent. Nine formulations Ch1, Ch2, Ch3, Ch4, Ch5, Ch6, Ch7, Ch8 and Ch9 were prepared successfully. The conc. of chitosan and TPP sod was varied to understand the behavior of Chitosan and TPP sod on particle size, entrapment efficiency and drug release behavior. Formulation Ch1 has the smallest size and good entrapment efficiency and good in-vitro drug release profile therefore $\mathrm{Ch} 1$ selected for further in-vivo study.

The pharmacokinetic study of $\mathrm{Ch} 1$ revealed that drug achieved $\mathrm{C}_{\max }$ in plasma in $45 \mathrm{~min}$ and it has taken only 5min to achieve the $\mathrm{C}_{\max }$ in brain whereas from i.v. route the $\mathrm{C}_{\max }$ in plasma and brain was achieved in $5 \mathrm{~min}$ and $30 \mathrm{~min}$ respectively. The drug targeting efficiency was found to be 1.735 , From the above study it was concluded that chitosan nanoparticles are able to transport the drug from nose to brain and can release the drug with enhance residence time and prolong drug release with lowest possible side effects due to lesser amount of drug reaches to the systemic circulation.

\section{ACKNOWLEDGEMENT:}

The authors are thankful to department of Pharmaceutical Sciences Bhimtal campus and also thankful to IIT Roorki for providing TEM and zeta sizing facilities. 


\section{REFERENCES}

1. Serralheiro A, Gilberto A, Ana F, Amílcar F, Intranasal administration of carbamazepine to mice: a direct delivery pathway for brain targeting, Euro. J. of Pharma Sci, 2014, doi:dx. doi.org/10. 1016/j.ejps. 2014. 04.019.

2. Gavini E, Hegge A B, Rassu G, Sanna V, Testa C, Pirisino G, Karlsen J, Giunchedi, P, Nasal administration of carbamazepine using chitosan microspheres: In-vitro/in-vivo studies. Int. J. Pharm, 2006, 307, 9-15.

3. Barakat N S, Omar S A, Ahmed A A E, Carbamazepine uptake into rat brain following intra-olfactory transport, J. of Pharmacology, 2006, 58: 63-72

4. Lee, V H, Robinson J R, Controlled drug delivery fundamental and application second edition, revised and expanded. Dekker. New York. 2005, 29, 4.

5. Ravi MNV, Handbook of Particulate drug delivery, American Sci publishers, California, vol 2, 96-161.

6. Chein YW, Novel drug delivery systems, 2nd Ed. New York, Marcel Dekker Inc; 1993, 29, 269-300.

7. Yildiz O, Particulate carrier for nasal administration, handbook of particulate drug delivery, American scientific publisher, 2008 2,143-161.

8. Sharma, P K, Garg G, Salim M, "Review on nasal drug delivery system with recent advancemnt", Int. J. Pharm. and Pharma. sci., 2011, 3, 6-11.

9. Sharma S, Lohan S, Murthy RSR, Potential of Chitosan For Nose To Brain Drug Delivery Int. J. Pharm. Sci. Rev. Res., 2012,16(1), 09, 47-55.

10. Ugwoke M I, Kinget V N, J. Pharm.pharmacol, 2001, 53, 3.

11. Calvo P, Lopez C R, Vila-Jato J L, Alonso M J, J Appll Polym Sci, 1997, 63:125-132.

12. Lokhandwala H, Deshpande A, Deshpande S, Kinetic modeling and dissolution profiles comparison: an overview, Int. J. Pharm. Bio. Sci., 2013 4(1),728 - 73

13. Dash S, Murthy P N, Nath L, Chowdhury P, Kinetic modeling on drug release from controlled, Drug delivery systems, Acta Polo. Pharm. Drug Rese, 2010, 67(3)217-223.

14. Chien, Nasal Drug Delivery and Delivery Systems, Drugs and the Pharma. Sci, 1991.

15. Sanghai B, Aggarwal G, HariKumar SL, Solid self microemulsifying drug deliviry system: a review, Journal of Drug Delivery and Therapeutics. 2013; 3(3):168-174

16. Jadhav K R, Manoj N, Gambhire, Shaikh I M, Nasal Drug Delivery System-Factors Affecting and Applications, Cur. Drug Therapy, 2007, 2, 27-38.

17. Banderas L M, "Nanostructures for Drug Delivery to the Brain", Cur. Med. Chem, 2011, 18(34), 5303-5321.

18. Mygind N, Dahl R, Anatomy and physiology of the nasal cavities in health and disease, adv. drug del. Rev, 1998, 29, 3 12.

19. Agnihotri S A, Mallikarjuna N N, Aminabhavi T M, Recent advances on chitosan-based micro-and nanoparticles in drug delivery. J of Contr. Rel, 2004,100(1), 5-28,

20. Agnieszka Z, Wilczewska, Katarzyna N, Karolina H. Markiewicz, Halina C, Nanoparticles as drug delivery systems, Pharmacological Reports, 2012, 64, 1020-1037.

21. Vyas S P, Khar R K, Targeted And Controlled Drug Delivery: Novel Carrier System, Vallabh Prakashan, Delhi,2010 reprint
22. Kaur I P, Bhandari R, Bhandari S, Kakkar V. Potential of solid lipid nanopartic les in brain targeting, J. of Contr. Rel, 2008, 127, 97-109.

23. Yadav G, Panchory H, Nanosponges: A Boon to the Targeted Drug Delivery System, Journal of Drug Delivery \& Therapeutics; 2013, 3(4), 151-155

24. Müller, R H, Mäder K, Gohla S, A review on SLNs for controlled drug delivery, Euro. J of Pharma. and Biopharma, 2000, 50(1),161-177.

25. Wong $\mathrm{H}$, Rauth A $\mathrm{M}$, Bendayan $\mathrm{R}, \mathrm{Wu} \mathrm{X} \mathrm{Y}$, In-vivo evaluation of a new polymer-lipid hybrid nanoparticles (PLN) formulation of doxorubicin in a murine solid tumor model, Euro. J of Pharma. and Biopharma, 2007, 65, 300-308.

26. Vyas, S P, Gowswami S K, Singh R, Liposamal based nasal delivery system of nifedipine: Delveloment and characterization, Int. J. Pharm, 1995, 118, 23-30.

27. Xiaoling G, Weixing T, Wei, L , Qizhi Z, Xianguo X Y, Lectin- conjugated nanoparticles: preparation and brain delivery after intranasal administration, Biomaterial, 2006, 27, 3, 482-490.

28. Soppimath, K S, Aminabhavi T M, Kulkarni A R, Rudzinski W E, Biodegradable polymeric nanoparticles as drug delivery devices. J. Con. Rel, 2001, 70, 1-20.

29. Packhaeuser C B, Schnieders J, Oster C G, Kissel T, In situ forming parenteral drug delivery systems: an overview, Eur. J. Pharm. Biopharm, 2004, 58, 445-55.

30. Nagai T, Nambu NY, powder dosage of insulin for nasal administration, J. Con. Rel, 1984, 1, 15-22.

31. Heiati H, Tawashi R, Phillips N C, Solid lipid nanoparticles as drug carriers II. Plasma stability and biodistribution of solid lipid nanoparticles containing the lipophilic prodrug 3\%-azido$3 \%$ deoxythymidine palmitate in mice, Int. J. Pharm, 1998, 174, 71-80.

32. Gizurarson S, Thorvaldsson T P, Sigurdsson G E, Selective delivery of insulin into the brain: Intraolfactory absorption, Int. J. Pharm vol, 1997, 146,135-141

33. Ikechukwa M, Ugwoke, Nasal mucoadhesive delivery of antiparkinson drug, apomorphine: influence of drug-loading on in-vitro and in-vivo release in rabbits Int. J. Pharma, 1999, 181(1)125-138.

34. Garg A, Gupta MM, Mouth dissolving tablets: a review, Journal of Drug Delivery and Therapeutics. 2013; 3(2):207214

35. Bagger M, Bechgaard E, A microdialysis model to examine nasal drug delivery and olfactory absorption in rats using lidocaine hydrochloride as a model drug. Int. J. Pharm, 2004, 269, 311-322.

36. Mascha P, Berg V D, Verhoef J C, Romeijn S G, Merkus F, Uptake of estradiol or progesterone into the CSF following intranasal and intravenous delivery in rats. Eur. J. Pharm. Biopharm, 2004, 58, 131-135.

37. Venkateswarlu V, Manjunath K, Preparation, characterization and in-vitro release kinetics of clozapine solid lipid nanoparticles, J. of Cont. Rel, 2004, 95, 627-638.

38. Carsten O, Lipid Drug Conjugate Nanoparticles of Hydrophilic Drug Diminazene- Cytotoxicity Testing and Mouse Serum Absorption, J. Con Rel.200418, 96(3)425-435. 